\title{
Biomarkers in Neurocritical Care
}

\author{
W. Taylor Kimberly
}

Published online: 22 November 2011

(C) The American Society for Experimental NeuroTherapeutics, Inc. 2011

\begin{abstract}
The gold standard for assessing neurological function is the bedside clinical examination. However, in neurocritical patients, the signs and symptoms related to the severity of illness can often be ambiguous. It can be hard to distinguish between a severe but stable disease state and one that is dynamic and in a critical decline. Clinicians and family members alike may struggle with the uncertainty of functional outcome prediction. Intermediate biomarkers of brain injury can assist with ongoing clinical management of patients, and in some circumstances can guide prognosis. Used in the right setting, biomarkers in neurocritical care can also aid with decisions to intensify treatment or avoid prolonged and unnecessary therapy. The term biomarker is used in various ways, and here we use it to refer to 3 general types: 1) circulating blood macromolecules, 2) brain imaging, and 3) continuous invasive monitors. Despite its promise, biomarkers have several limitations and should be interpreted in the context of the overall clinical assessment.
\end{abstract}

Keywords Neurocritical care · Biomarker - Surrogate endpoint $\cdot$ Neuroimaging $\cdot$ Intracranial monitor.

\section{Introduction}

There are many challenges to the diagnosis and management of patients with neurocritical illness. Although serial

\footnotetext{
W. T. Kimberly $(\triangle)$

Department of Neurology,

Division of Neurocritical Care and Emergency Neurology,

Massachusetts General Hospital,

Boston, MA 02114, USA

e-mail: wtkimberly@partners.org
}

neurological exams are a cornerstone of care, the severity of disease often obscures neurological assessments in the patients with acute brain injury. In this setting, biomarkers can act as a surrogate measure and may serve a variety of capacities. They may assist in diagnosis or help monitor a response to treatment. In some circumstances, biomarkers can help clarify the severity of disease or can provide risk stratification and risk prediction. Equally important, biomarkers can serve to guide treatment decisions in a dynamic clinical situation [1]. However, in practice individual biomarkers rarely replace other elements of clinical assessment, but are better viewed as adjuncts to decision making (Table 1).

In general, biomarkers tend to be more informative the more closely related they are to the underlying pathogenesis of the disease. Yet even in that setting, it can be difficult to dissociate whether a given biomarker is causally linked to outcome or simply associated with it. The aim of this review is to provide an outline of the types, strengths, and limitations of biomarkers used in neurocritical care.

\section{A Common Endpoint in Neurocritical Care}

While there are varied types of acute brain injuries in the neurocritical care setting, a unifying goal is the prevention of secondary neurological injury. Secondary insults take numerous forms, including elevated intracranial pressure, brain edema, impaired tissue perfusion, disordered cerebrovascular autoregulation, and focal tissue shifts from mass lesions. In each of these cases, therapeutic interventions are designed to support brain tissue at risk and ultimately prevent brain energetic failure. Many biomarkers aim to measure some aspects of brain metabolism or quantify the degree of brain injury. Given the diversity of insults that threaten the brain, it is unlikely that any single biomarker is capable of acting as a universal surrogate. 
Table 1 A summary of the types of biomarkers used in neurocritical care

\begin{tabular}{|c|c|c|c|}
\hline Neuroimaging & Blood & Invasive & Other \\
\hline Intracranial hypertension & & ICP monitor & \\
\hline \multirow[t]{3}{*}{ Stroke } & \multirow[t]{3}{*}{ Head CT } & MMP-9 & \\
\hline & & Brain MRI & S100-beta \\
\hline & & c-fibronectin & \\
\hline \multirow[t]{2}{*}{ Intracerebral hemorrhage } & Head CT & & \\
\hline & Head CT angiography & & \\
\hline \multirow[t]{4}{*}{ Traumatic brain injury } & Head CT & S100-beta & ICP monitor \\
\hline & Brain MRI & GFAP & $\mathrm{PbO}_{2}$ \\
\hline & SWI, DWI, MRS & UCH-L1 & Microdialysis \\
\hline & FLAIR, DTI & $\mathrm{SjO}_{2}$ & \\
\hline \multirow[t]{2}{*}{ SAH vasospasm } & Cerebral angiography & $\mathrm{PbO}_{2}$ & TCDs \\
\hline & Head CT angiography & Microdialysis & EEG \\
\hline
\end{tabular}

$\mathrm{CT}=$ computed tomography; DTI = diffusion tensor imaging; DWI = diffusion weighted imaging; EEG = electroencephalography; FLAIR = fluid attenuated inversion recovery; GFAP = glial fibrillary acid protein; ICP = intracranial pressure; MMP-9 = matrix metalloproteinase 9; MRI = magnetic resonance imaging; MRS = magnetic resonance spectroscopy; SAH $=$ subarachnoid hemorrhage; SWI $=$ suscetibility weight imaging; TCDs $=$ transcranial Doppler ultrasounds; UCH-L1 = ubiquitin C-terminal hydrolase L1 protein

\section{Elevated Intracranial Pressure}

Perhaps the most recognizable neurocritical emergency is the acute elevation of intracranial pressure (ICP). Once ICP reaches a critical threshold, it prevents adequate blood flow and impairs cerebral perfusion, which in turn leads to irreversible ischemia. Therefore, invasive ICP monitors play a central role in the management of patients suspected of having elevated ICP. These devices not only give diagnostic information, but the continuous measurement can guide targeted therapy thereafter. In addition to measuring the ICP level itself, the cerebral perfusion pressure (CPP) can also be followed if the patient has an arterial line. CPP is a reflection of the pressure gradient between the systemic and cerebral circulation, and indirectly provides a measure of cerebral blood flow. It can be readily derived from the equation: $\mathrm{CPP}=$ mean arterial pressure-ICP. In this way, ICP and CPP measurements are a surrogate for adequate brain perfusion and for sustaining the bio-energetic homeostasis of the brain. An ICP of $20 \mathrm{mmHg}$ is a common threshold for treatment. Available animal studies suggest that an important CPP threshold exists at approximately $50 \mathrm{mmHg}$ [2]. ICP- and CPP-guided therapy is a useful tool in neurocritical care, because decisions as to treatment with osmotherapy or potentially surgical management can be made in real time, based on continuous monitoring.

As central as ICP measurement is for clinical management, the optimal ICP and CPP thresholds are not necessarily fixed. In many pathological circumstances, the appropriate ICP target level may vary geographically and chronologically. For example, traumatic brain injury results in regionally varied sensitivity to blood flow [3], and a CPP target of $>50 \mathrm{mmHg}$ may be too low $[4,5]$. Another example is the use of hyperventilation in ICP management. Acute reductions in arterial $\mathrm{PaCO}_{2}$ through hyperventilation result in a rapid decrease in ICP. However, this is achieved by inducing cerebral arterial vasoconstriction, which can deprive the injured brain of much-needed arterial blood flow. In traumatic brain injury, this may paradoxically lead to brain oxygen desaturation [6] and a worse outcome [7], demonstrating how targeting ICP therapy to a threshold can be an imperfect surrogate for brain energy homeostasis.

\section{Ischemic Stroke}

In ischemic stroke, secondary neurological decline can occur as a result of either hemorrhagic complication of thrombolysis or malignant cerebral edema. Symptomatic hemorrhage, defined as a decline in the National Institutes of Health Stroke Scale (NIHSS) score of $\geq 4$ in the setting of a new hemorrhage [8], occurs in approximately $5 \%$ of patients treated with intravenous recombinant tissue plasminogen activator (IV t-PA) [9, 10]. By definition, this presents within the first 36 hours of treatment and is the most feared complication of IV t-PA. Malignant cerebral edema is associated with large hemispheric strokes in relatively young patients [11], and ensues within 48 to 72 hours of stroke onset. For each of these conditions, there are neuroimaging and blood-based biomarkers used for risk stratification and clinical decision making. 
Hemorrhagic Transformation

Neuroimaging biomarkers for hemorrhagic conversion include computed tomography (CT) and magnetic resonance imaging (MRI). Head CT assessment at 24 hours is the standard of care after thrombolysis, and detects hemorrhage in approximately $11 \%$ of patients $[9,10]$. CT detects two types of hemorrhage, termed hemorrhagic infarction and parenchymal hematoma [12]. On the one hand, petechial hemorrhagic infarction is associated with increased rates of recanalization and improved outcomes $[13,14]$. On the other, parenchymal hematoma is associated with worse outcomes $[15,16]$, particularly if it causes mass effect. Brain MRI has also been studied as a neuroimaging biomarker for hemorrhagic transformation, and its sensitivity for the detection of petechial hemorrhage is increased compared to CT. However, there is no difference in the detection of the more clinically significant parenchymal hematoma [17].

Current evidence suggests that parenchymal hemorrhage results from an increased disruption of the blood brain barrier [18, 19]. Matrix metalloproteinase 9 (MMP-9) is a proteolytic enzyme that degrades basement membrane components, including laminin and fibronectin, which are 2 major constituents of the blood brain barrier [20]. Therefore, MMP-9 has been studied as a blood-based biomarker for risk stratification in symptomatic hemorrhage. Measurement of baseline MMP-9 prior to IV t-PA treatment demonstrates that a higher baseline level is associated with increased risk of subsequent parenchymal hematoma [20-22]. Using a cutoff value of $\geq 140 \mathrm{ng} / \mathrm{ml}, 1$ study found there was $92 \%$ sensitivity and $74 \%$ specificity for detecting parenchymal hematoma [21]. However, to develop this for clinical utility, a rapid method would be needed to assist with clinical decision making and/or triaging.

\section{Malignant Cerebral Edema}

Malignant cerebral edema develops in approximately $10 \%$ of ischemic strokes, and has a mortality rate that exceeds $50 \%$ [11]. The only treatment shown to improve outcome is decompressive craniectomy [23], but it can be difficult to predict which patients will develop a malignant course that would necessitate surgery. Neuroimaging biomarkers for malignant edema include early hypodensity on CT, involvement of $>50 \%$ of the MCA territory [24] and midline shift $>5 \mathrm{~mm}$ within the first 48 hours [25]. However, the positive predictive value of these imaging surrogates is insufficient to prompt a decision for surgery in the absence of clinical deterioration. More recently, MRIbased imaging biomarkers for edema have been developed to predict early neurological deterioration. The percent of hemispheric growth on the side of the lesion can predict neurological deterioration with an area under the curve of 0.92 (K. Sheth, personal communication).

Blood-based biomarkers that correlate with size of infarction, such as $\mathrm{S100B}$, can help predict which patients will be at higher risk of developing a malignant syndrome. $\mathrm{S} 100 \mathrm{~B}$ is a glial-derived protein that is released by astrocytes in proportion to the degree of brain injury [26]. One study found a $24-\mathrm{h} \mathrm{S100B}$ value $>1.03 \mathrm{ug} / 1$ provided a sensitivity of $94 \%$ and a specificity of $83 \%$ for the development of malignant edema [27]. A second protein that is a constituent of the blood brain barrier (cellular fibronectin) had a sensitivity of $90 \%$ and a specificity of $100 \%$ for the prediction of a malignant course of infarction when a cutoff value $>16.6 \mathrm{ug} / \mathrm{ml}$ was used $[28,29]$.

\section{Intracranial Hemorrhage}

Intracerebral hemorrhage is a particularly deadly form of stroke, with a mortality of $33 \%$ at 3 months and $60 \%$ at 1 year [30]. The prevention of hematoma expansion is a major focus of medical management, and expansion is quite common, being observed in $40 \%$ of patients who present within the first 3 hours of onset [31-33]. Several ongoing clinical trials are aimed at the prevention of hematoma expansion, either through blood pressure management or hemostatic therapy. However, the complexity of using hematoma expansion on head CT as a surrogate biomarker was recently illustrated by the factor VIIa trial. Although a statistically significant decrease in hematoma expansion was demonstrated with treatment, there was no difference in clinical outcome [34].

More recently, the presence of ongoing hemorrhage detected on contrast-enhanced head CT ("spot sign") has been recognized as a more refined imaging biomarker for continued hematoma expansion [35, 36]. Current efforts in treating intracerebral hemorrhage in the acute setting are focused on patients in whom a spot sign is present, although the efficacy of those therapies is under active investigation.

\section{Traumatic Brain Injury}

Severe traumatic brain injury (TBI) is a major cause of death and disability, particularly in the young population. A major challenge in the clinical management of TBI is its heterogeneity, reflected by the myriad classification schemes in use. For example, classification can be based on clinical severity (i.e., Glasgow coma score), physical mechanism or pathoanatomic subtype [37]. Pathoanatomic classification is most often used for acute management in 
the neurocritical care setting, and includes epidural, subdural, and subarachnoid traumatic hemorrhages, parenchymal contusion, diffuse axonal injury, and cerebral edema [37]. Several neuroimaging and invasive biomarkers assist clinicians in acute management at the time when secondary injury can occur. Other biomarkers are helpful in refining long-term prognosis, many of which are under active investigation.

\section{Biomarkers for Acute Management}

After the acute trauma, severe TBI patients are at high risk for developing subsequent injury. This may result from global or focal increased intracranial pressure. The correlation between elevated ICP and poor outcomes in severe TBI is well-described [38-40], and comatose patients with a glasgow coma score (GCS) $<9$ are at highest risk for developing high ICP [41]. Although the placement of an intracranial pressure monitor in severe TBI is nearly universal, ICP- and CPP-targeted therapy has not formally been shown to improve outcomes in randomized controlled trials [42, 43]. Nevertheless, in patients who respond to therapy that successfully lowers ICP, better outcomes have been observed [44, 45]. Treatment can be targeted to an ICP threshold, a CPP threshold, or both. However, the appropriate thresholds may vary from patient to patient $[46,47]$.

Because ICP and CPP do not perfectly reflect brain physiology and energy homeostasis, brain tissue oxygen monitoring has emerged as a complementary tool. Brain oxygenation can be measured by jugular venous oxygen saturation $\left(\mathrm{SjO}_{2}\right)$ [48] or by brain tissue oxygen tension $\left(\mathrm{PbO}_{2}\right)$ [49]. Lower $\mathrm{SjO}_{2}$ values $(<50 \%)$ or $\mathrm{PbO}_{2}$ values less than $15 \mathrm{mmHg}$ have been associated with worse outcomes [50]. Given the correlation of lower oxygen values with poor outcome, it raises the question whether treatment directed at increasing those values can improve outcomes. One nonblinded, nonrandomized study has suggested that $\mathrm{SjO}_{2}$ targeted treatment may improve outcome [51]. Similarly, $\mathrm{PbO}_{2}$-targeted therapy with a comparison to historical controls supports the notion that treatment to a threshold can improve outcome [49]. However, definitive conclusions are limited due to the lack of randomized trials, and this has led to substantial centerto-center variation in the implementation of these tools.

Another biomarker designed to assess brain energy homeostasis is cerebral microdialysis [52]. This bedside diagnostic tool has the capability to assess energy metabolism of the extracellular fluid of the brain through frequent measurements of the lactate/pyruvate ratio, glycerol (a measure of degeneration), and glutamate (as a measure of excitotoxicity). Biochemical changes in the lactate/pyruvate ratio (i.e., a ratio $>25$ ) have been reported to occur prior to detecting elevated ICP or low CPP [53] and also predict poor outcome in TBI [54]. However, it remains to be determined whether tailoring treatment to certain target levels can influence outcome.

\section{Biomarkers for Prognosis}

Prognosis in the acute setting of TBI is often imprecise, yet discussions as to whether to proceed with aggressive supportive care are frequently made early in the course of care. Existing clinical prognostic tools, based on age, pupil reactivity, and initial GCS [55] do not provide the level of detail that many families seek in their decision-making [26]. Several neuroimaging and blood proteins are used as prognostic biomarkers. Using the Marshall classification, the presence of significant brain swelling on head CT predicts poor outcome [56]. Further refinement on the classification of abnormalities on admission head CT have suggested that presence of subarachnoid blood, "massive" focal, or diffuse injury are independent prognostic variables for death [57]. More recently, advancement in brain MRIs has revealed diffuse axonal injury that is not visible on a routine head CT, which is associated with a degree of functional outcome rather than mortality. For example, lesion volumes on fluid attenuated inversion recovery (FLAIR), susceptibility weighted imaging, and diffusionweighted imaging all demonstrate a significant correlation with 6-month outcome [58, 59]. Similarly, magnetic resonance spectroscopy is capable of detecting spatial variations in several markers of neuronal and axonal health, including creatine, choline, and $\mathrm{N}$-acetyl aspartate. Magnetic resonance spectroscopy has been shown to improve prediction of 6-month outcome, particularly when a ratio of choline to creatine is used [60]. Finally, diffusion tensor imaging is a relatively new tool that can assess patency of the white matter tracts of the brain [61]. These tools, while improving the accuracy of functional outcome prediction, may not be easily implemented in all centers.

Several blood-based biomarkers have also shown some promise as prognostic measures for outcome in TBI. S100beta is a protein in glial cells that when elevated can predict poor outcome [62], particularly around a threshold of $2.0 \mathrm{ug} / \mathrm{l}$. However, S100B expression is not limited to the central nervous system, and it can be elevated in the setting of significant extra-cranial injuries which are common in TBI patients [26]. Glial fibrillary acid protein is a brain-specific protein that is expressed in high levels in astrocyte; it is released in the blood after acute brain injury and has been reported to predict clinical outcome and mortality [63, 64]. Finally, ubiquitin C-terminal hydrolase L1 protein is specifically expressed in neurons and is also released into the bloodstream after injury. The levels of these proteins not only correlate with the severity of TBI, but they also predict mortality at 6 months [65]. 


\section{Vasospasm After Subarachnoid Hemorrhage}

Aneurysmal subarachnoid hemorrhage (SAH) has a high mortality rate and poor outcome, with more than half of the patients experiencing death or dependency [66, 67]. Those who survive the initial hemorrhage face the risk of further secondary injury, which results in additional disability. Delayed cerebral ischemia (DCI), is a leading cause of subsequent neurological injury [68]. DCI overlaps with vasospasm, but is also distinct from it; vasospasm is a radiographic descriptor of abnormal cerebral arterial narrowing. Because poor grade SAH patients can experience DCI and/or vasospasm in the absence of detectable neurological deterioration, surrogate measures are needed. Often, biomarkers for DCI and vasospasm are used interchangeably, even though one may occur without the other. Aside from frequent neurological examinations, accurate detection of DCI relies on head CT and MRI. CT can detect clinically asymptomatic infarcts, particularly in patients with low GCS $[69,70]$. MRI can detect a larger proportion of asymptomatic infarcts compared to CT [71], and importantly the overall burden of infarcts is correlated with outcome [72, 73].

For the detection of vasospasm, several modalities are used, including digital subtraction angiography, CT angiography and transcranial Doppler ultrasound. Emerging biomarkers include electroencephalography and invasive cerebral monitoring of $\mathrm{PbO}_{2}$ and microdialysis. Digital subtraction angiography (DSA) is considered the gold standard for detection of vasospasm, and CT angiography has a high correlation with DSA arterial narrowing [74, 75]. Transcranial Doppler ultrasounds are frequently used for daily monitoring, but have less sensitivity compared to DSA [76]. Electroencephalography monitoring of alpha variability has shown correlation with vasospasm and clinically significant ischemia in preliminary studies [77]. Similar observational trials with $\mathrm{PbO}_{2}$ monitoring [78] and microdialysis [79] have shown feasibility of these techniques, but no studies have demonstrated efficacy of treatment based on these monitors. Taken together, modalities for the monitoring of vasospasm are an important part of the care of SAH patients, but further work is needed to determine whether targeting treatment of the abnormalities influences clinical outcome.

\section{Conclusions}

Although biomarkers can help reduce uncertainty as to neurocritical disease and its prognosis, no single biomarker is universally suitable for all clinical situations. Rather, the incorporation of salient biomarkers into the overall clinical assessment will continue to be an important tool for neurointensivists. Because many biomarkers address com- plementary aspects of brain energy homeostasis and brain injury, it is likely that multimodal measurements will increasingly become a part of practice. A major challenge in moving forward will be to validate current biomarkers as surrogate endpoints and to demonstrate that treatment decisions based on abnormal values can influence outcome. Multi-institution collaborations will be a necessity to adequately address these questions and advance the care of patients with neurocritical illness.

Acknowledgments This work was supported by the National Institutes of Health (No. 1K23NS076597) and a grant from the Clinical Investigator Training Program: Beth Israel Deaconess Medical Center, Harvard Medical School, in collaboration with Pfizer Inc. and Merck \& Co.

Required Author Forms Disclosure forms provided by the authors are available with the online version of this article.

\section{References}

1. Atkinson AJ, Colburn WA, DeGruttola VG, et al. Biomarkers and surrogate endpoints: preferred definitions and conceptual framework. Clin Pharmacol Ther 2001;69:89-95.

2. Vespa P. What is the optimal threshold for cerebral perfusion pressure following traumatic brain injury? Neurosurg Focus 2003; 15:E4.

3. Cunningham AS, Salvador R, Coles JP, et al. Physiological thresholds for irreversible tissue damage in contusional regions following traumatic brain injury. Brain 2005;128:1931-1942.

4. Robertson CS, Valadka AB, Hannay HJ, et al. Prevention of secondary ischemic insults after severe head injury. Crit Care Med 1999;27:2086-2095.

5. Rosner MJ, Rosner SD, Johnson AH. Cerebral perfusion pressure: management protocol and clinical results. J Neurosurg 1995;83:949-962.

6. Gopinath SP, Robertson CS, Contant CF, et al. Jugular venous desaturation and outcome after head injury. J Neurol Neurosurg Psychiatry 1994;57:717-723.

7. Muizelaar JP, Marmarou A, Ward JD, et al. Adverse effects of prolonged hyperventilation in patients with severe head injury: a randomized clinical trial. J Neurosurg 1991;75:731-739.

8. Furlan A, Higashida R, Wechsler L, et al. Intra-arterial prourokinase for acute ischemic stroke. The proact II study: a randomized controlled trial. Prolyse in acute cerebral thromboembolism. JAMA 1999;282:2003-2011.

9. The National Institute of Neurological Disorders and Stroke rt-PA Stroke Study Group. Tissue plasminogen activator for acute ischemic stroke. N Engl J Med 1995;333:1581-1587.

10. Graham GD. Tissue plasminogen activator for acute ischemic stroke in clinical practice: a meta-analysis of safety data. Stroke 2003;34:2847-2850.

11. Kimberly WT, Sheth KN. Approach to severe hemispheric stroke. Neurology 2011;76:S50-S56.

12. Hacke W, Kaste M, Fieschi C, et al. Intravenous thrombolysis with recombinant tissue plasminogen activator for acute hemispheric stroke. The European Cooperative Acute Stroke Study (ECASS). JAMA 1995;274:1017-1025.

13. Molina CA, Alvarez-Sabin J, Montaner J, et al. Thrombolysis-related hemorrhagic infarction: a marker of early reperfusion, reduced infarct 
size, and improved outcome in patients with proximal middle cerebral artery occlusion. Stroke 2002;33:1551-1556.

14. Fiehler J, Remmele C, Kucinski T, et al. Reperfusion after severe local perfusion deficit precedes hemorrhagic transformation: an MRI study in acute stroke patients. Cerebrovasc Dis 2005;19:117-124.

15. Berger C, Fiorelli M, Steiner T, et al. Hemorrhagic transformation of ischemic brain tissue: asymptomatic or symptomatic? Stroke 2001;32:1330-1335.

16. Larrue V, von Kummer RR, Muller A, Bluhmki E. Risk factors for severe hemorrhagic transformation in ischemic stroke patients treated with recombinant tissue plasminogen activator: a secondary analysis of the European-Australasian Acute Stroke Study (ECASS II). Stroke 2001;32:438-441.

17. Thomalla G, Sobesky J, Kohrmann M, et al. Two tales: hemorrhagic transformation but not parenchymal hemorrhage after thrombolysis is related to severity and duration of ischemia: MRI study of acute stroke patients treated with intravenous tissue plasminogen activator within 6 hours. Stroke 2007;38:313-318.

18. Sumii T, Lo EH. Involvement of matrix metalloproteinase in thrombolysis-associated hemorrhagic transformation after embolic focal ischemia in rats. Stroke 2002;33:831-836.

19. Wang X, Tsuji K, Lee SR, et al. Mechanisms of hemorrhagic transformation after tissue plasminogen activator reperfusion therapy for ischemic stroke. Stroke 2004;35:2726-2730.

20. Ning M, Furie KL, Koroshetz WJ, et al. Association between tpa therapy and raised early matrix metalloproteinase-9 in acute stroke. Neurology 2006;66:1550-1555.

21. Castellanos M, Sobrino T, Millan M, et al. Serum cellular fibronectin and matrix metalloproteinase- 9 as screening biomarkers for the prediction of parenchymal hematoma after thrombolytic therapy in acute ischemic stroke: a multicenter confirmatory study. Stroke 2007;38:1855-1859.

22. Montaner J, Molina CA, Monasterio J, et al. Matrix metalloproteinase9 pretreatment level predicts intracranial hemorrhagic complications after thrombolysis in human stroke. Circulation 2003;107:598-603.

23. Vahedi K, Hofmeijer J, Juettler E, et al. Early decompressive surgery in malignant infarction of the middle cerebral artery: A pooled analysis of three randomised controlled trials. Lancet Neurol 2007;6:215-222.

24. Kasner SE, Demchuk AM, Berrouschot J, et al. Predictors of fatal brain edema in massive hemispheric ischemic stroke. Stroke 2001;32:2117-2123.

25. Pullicino PM, Alexandrov AV, Shelton JA, Alexandrova NA, Smurawska LT, Norris JW. Mass effect and death from severe acute stroke. Neurology 1997;49:1090-1095.

26. Bloomfield SM, McKinney J, Smith L, Brisman J. Reliability of $\mathrm{s} 100 \mathrm{~b}$ in predicting severity of central nervous system injury. Neurocrit Care 2007;6:121-138

27. Foerch C, Otto B, Singer OC, et al. Serum s100b predicts a malignant course of infarction in patients with acute middle cerebral artery occlusion. Stroke 2004;35:2160-2164.

28. Castellanos M, Leira R, Serena J, et al. Plasma cellularfibronectin concentration predicts hemorrhagic transformation after thrombolytic therapy in acute ischemic stroke. Stroke 2004;35:1671-1676.

29. Serena J, Blanco M, Castellanos M, et al. The prediction of malignant cerebral infarction by molecular brain barrier disruption markers. Stroke 2005;36:1921-1926.

30. Qureshi AI, Mendelow AD, Hanley DF. Intracerebral haemorrhage. Lancet 2009;373:1632-1644.

31. Brott T, Broderick J, Kothari R, et al. Early hemorrhage growth in patients with intracerebral hemorrhage. Stroke 1997;28:1-5.

32. Flibotte JJ, Hagan N, O'Donnell J, Greenberg SM, Rosand J. Warfarin, hematoma expansion, and outcome of intracerebral hemorrhage. Neurology 2004;63:1059-1064.
33. Davis SM, Broderick J, Hennerici M, et al. Hematoma growth is a determinant of mortality and poor outcome after intracerebral hemorrhage. Neurology 2006;66:1175-1181.

34. Mayer SA, Brun NC, Begtrup K, et al. Efficacy and safety of recombinant activated factor vii for acute intracerebral hemorrhage. N Engl J Med 2008;358:2127-2137.

35. Wada R, Aviv RI, Fox AJ, et al. Ct angiography "spot sign" predicts hematoma expansion in acute intracerebral hemorrhage. Stroke 2007;38:1257-1262.

36. Becker KJ, Baxter AB, Bybee HM, Tirschwell DL, Abouelsaad T, Cohen WA. Extravasation of radiographic contrast is an independent predictor of death in primary intracerebral hemorrhage. Stroke 1999;30:2025-2032.

37. Saatman KE, Duhaime AC, Bullock R, Maas AI, Valadka A, Manley GT. Classification of traumatic brain injury for targeted therapies. J Neurotrauma 2008;25:719-738.

38. Becker DP, Miller JD, Ward JD, Greenberg RP, Young HF, Sakalas R. The outcome from severe head injury with early diagnosis and intensive management. J Neurosurg 1977;47:491-502.

39. Lundberg N, Troupp H, Lorin H. Continuous recording of the ventricular-fluid pressure in patients with severe acute traumatic brain injury. A preliminary report. J Neurosurg 1965;22:581-590.

40. Narayan RK, Greenberg RP, Miller JD, et al. Improved confidence of outcome prediction in severe head injury. A comparative analysis of the clinical examination, multimodality evoked potentials, CT scanning, and intracranial pressure. J Neurosurg 1981;54:751-762.

41. Narayan RK, Kishore PR, Becker DP, et al. Intracranial pressure: to monitor or not to monitor? A review of our experience with severe head injury. J Neurosurg 1982;56:650-659.

42. Palmer S, Bader MK, Qureshi A, et al. The impact on outcomes in a community hospital setting of using the aans traumatic brain injury guidelines. American Association for Neurologic Surgeons. J Trauma 2001;50:657-664.

43. Fakhry SM, Trask AL, Waller MA, Watts DD. Management of brain-injured patients by an evidence-based medicine protocol improves outcomes and decreases hospital charges. J Trauma 2004;56:492-500.

44. Eisenberg HM, Frankowski RF, Contant CF, Marshall LF, Walker MD. High-dose barbiturate control of elevated intracranial pressure in patients with severe head injury. J Neurosurg 1988;69:15-23

45. Saul TG, Ducker TB. Effect of intracranial pressure monitoring and aggressive treatment on mortality in severe head injury. J Neurosurg 1982;56:498-503.

46. Bratton SL, Chestnut RM, Ghajar J, et al. Guidelines for the management of severe traumatic brain injury. Ix. Cerebral perfusion thresholds. J Neurotrauma 2007;24(suppl 1):S59-S64.

47. Bratton SL, Chestnut RM, Ghajar J, et al. Guidelines for the management of severe traumatic brain injury. Viii. Intracranial pressure thresholds. J Neurotrauma 2007;24(suppl 1):S55-S58.

48. Robertson CS, Gopinath SP, Goodman JC, Contant CF, Valadka AB, Narayan RK. Sjvo2 monitoring in head-injured patients. J Neurotrauma 1995;12:891-896.

49. Stiefel MF, Spiotta A, Gracias VH, et al. Reduced mortality rate in patients with severe traumatic brain injury treated with brain tissue oxygen monitoring. J Neurosurg 2005;103:805-811.

50. Valadka AB, Gopinath SP, Contant CF, Uzura M, Robertson CS Relationship of brain tissue $\mathrm{PO} 2$ to outcome after severe head injury. Crit Care Med 1998;26:1576-1581.

51. Cruz J. The first decade of continuous monitoring of jugular bulb oxyhemoglobinsaturation: management strategies and clinical outcome. Crit Care Med 1998;26:344-351.

52. Tisdall MM, Smith M. Cerebral microdialysis: research technique or clinical tool. Br J Anaesth 2006;97:18-25. 
53. Belli A, Sen J, Petzold A, Russo S, Kitchen N, Smith M. Metabolic failure precedes intracranial pressure rises in traumatic brain injury: a microdialysis study. Acta Neurochir (Wien) 2008;150:461-470.

54. Timofeev I, Carpenter KL, Nortje J, et al. Cerebral extracellular chemistry and outcome following traumatic brain injury: a microdialysis study of 223 patients. Brain 2011;134:484-494.

55. Signorini DF, Andrews PJ, Jones PA, Wardlaw JM, Miller JD. Predicting survival using simple clinical variables: A case study in traumatic brain injury. J Neurol Neurosurg Psychiatry 1999;66:20-25.

56. Eisenberg HM, Gary HE Jr, Aldrich EF, et al. Initial ct findings in 753 patients with severe head injury. A report from the nih traumatic coma data bank. J Neurosurg 1990;73:688-698.

57. Wardlaw JM, Easton VJ, Statham P. Which ct features help predict outcome after head injury? J Neurol Neurosurg Psychiatry 2002;72:188-192.

58. Marquez de la Plata C, Ardelean A, Koovakkattu D, et al. Magnetic resonance imaging of diffuse axonal injury: quantitative assessment of white matter lesion volume. J Neurotrauma 2007;24:591-598.

59. Babikian T, Freier MC, Tong KA, et al. Susceptibility weighted imaging: Neuropsychologic outcome and pediatric head injury. Pediatr Neurol 2005;33:184-194.

60. Shutter L, Tong KA, Holshouser BA. Proton mrs in acute traumatic brain injury: role for glutamate/glutamine and choline for outcome prediction. J Neurotrauma 2004;21:1693-1705.

61. Warner MA, Marquez de la Plata C, Spence J, et al. Assessing spatial relationships between axonal integrity, regional brain volumes, and neuropsychological outcomes after traumatic axonal injury. J Neurotrauma 2010;27:2121-2130.

62. Petzold A, Green AJ, Keir G, et al. Role of serum s100b as an early predictor of high intracranial pressure and mortality in brain injury: a pilot study. Crit Care Med 2002;30:27052710.

63. Vos PE, Lamers KJ, Hendriks JC, et al. Glial and neuronal proteins in serum predict outcome after severe traumatic brain injury. Neurology 2004;62:1303-1310.

64. Raabe A, Grolms C, Keller M, Dohnert J, Sorge O, Seifert V. Correlation of computed tomography findings and serum brain damage markers following severe head injury. Acta Neurochir (Wien) 1998;140:787-791.

65. Mondello S, Papa L, Buki A, et al. Neuronal and glial markers are differently associated with computed tomography findings and outcome in patients with severe traumatic brain injury: a case control study. Crit Care 2011;15:R156.

66. Pierot L, Aggour M, Moret J. Vasospasm after aneurysmal subarachnoid hemorrhage: recent advances in endovascular management. Curr Opin Crit Care 2010;16:110-116.
67. Longstreth WT Jr., Nelson LM, Koepsell TD, van Belle G. Clinical course of spontaneous subarachnoid hemorrhage: a population-based study in king county, washington. Neurology 1993;43:712-718.

68. Macdonald RL, Pluta RM, Zhang JH. Cerebral vasospasm after subarachnoid hemorrhage: the emerging revolution. Nat Clin Pract Neurol 2007;3:256-263.

69. Rabinstein AA, Weigand S, Atkinson JL, Wijdicks EF. Patterns of cerebral infarction in aneurysmal subarachnoid hemorrhage. Stroke 2005;36:992-997.

70. Schmidt JM, Wartenberg KE, Fernandez A, et al. Frequency and clinical impact of asymptomatic cerebral infarction due to vasospasm after subarachnoid hemorrhage. J Neurosurg 2008;109:1052-1059.

71. Shimoda M, Takeuchi M, Tominaga J, Oda S, Kumasaka A, Tsugane R. Asymptomatic versus symptomatic infarcts from vasospasm in patients with subarachnoid hemorrhage: serial magnetic resonance imaging. Neurosurgery 2001;49:1341-1350.

72. Vergouwen MD, Etminan N, Ilodigwe D, Macdonald RL. Lower incidence of cerebral infarction correlates with improved functional outcome after aneurysmal subarachnoid hemorrhage. J Cereb Blood Flow Metab 2011;31:1545-1553.

73. Kreiter KT, Mayer SA, Howard G, et al. Sample size estimates for clinical trials of vasospasm in subarachnoid hemorrhage. Stroke 2009;40:2362-2367.

74. Yoon DY, Choi CS, Kim KH, Cho BM. Multidetector-row ct angiography of cerebral vasospasm after aneurysmal subarachnoid hemorrhage: comparison of volume-rendered images and digital subtraction angiography. AJNR Am J Neuroradiol 2006;27:370-377.

75. Chaudhary SR, Ko N, Dillon WP, et al. Prospective evaluation of multidetector-row ct angiography for the diagnosis of vasospasm following subarachnoid hemorrhage: a comparison with digital subtraction angiography. Cerebrovasc Dis 2008;25:144-150.

76. Lysakowski C, Walder B, Costanza MC, Tramer MR. Transcranial doppler versus angiography in patients with vasospasm due to a ruptured cerebral aneurysm: a systematic review. Stroke 2001;32:2292-2298.

77. Claassen J, Mayer SA, Hirsch LJ. Continuous eeg monitoring in patients with subarachnoid hemorrhage. J Clin Neurophysiol 2005;22:92-98.

78. Jaeger M, Schuhmann MU, Soehle M, Nagel C, Meixensberger J. Continuous monitoring of cerebrovascular autoregulation after subarachnoid hemorrhage by brain tissue oxygen pressure reactivity and its relation to delayed cerebral infarction. Stroke 2007;38:981-986.

79. Unterberg AW, Sakowitz OW, Sarrafzadeh AS, Benndorf G, Lanksch WR. Role of bedside microdialysis in the diagnosis of cerebral vasospasm following aneurysmal subarachnoid hemorrhage. J Neurosurg 2001;94:740-749. 